\title{
MOISTURE INDUCED DEGRADATION OF POROUS LOW-K MATERIALS
}

\author{
M. R. Baklanov, D. O’Dwyer, A. M. Urbanowicz ${ }^{1}$, Q. T. Le, S. Demuynck, and E. Hong². \\ IMEC, Leuven, Belgium \\ ${ }^{1}$ Wroclaw University of Technology, Poland \\ ${ }^{2}$ Assignee at IMEC from SAMSUNG Electronics.
}

\begin{abstract}
Interaction of moisture with porous low-k films is evaluated by using in situ ellipsometry setup. The adsorbed water amount is calculated from change of refractive index measured during the adsorption. Pristine low-k films reversibly adsorb 2 - 5\% of water that reflects presence of constitutive hydrophilic centrums. Plasma and thermal treatments increase the number of hydrophilic centrums. Once the amount of these centrums has reached a certain critical value sufficient to form a continuous water film, bulk water condensation is observed. Change of properties during the water adsorption in the damaged films is not fully reversible. Each additional adsorption cycle increases the dielectric function of the film because of decreasing porosity, increasing skeleton density and shrinkage. The pressure corresponding to the bulk condensation allows us to calculate internal contact angle (internal surface energy) of low-k materials. The water molecules adsorbed on separate $\mathrm{OH}$ groups play the role of a catalyst that hydrolyses the siloxane bridges initially present on hydrophobic surface.
\end{abstract}

\section{INTRODUCTION}

One of the most difficult problems during the integration of porous low-k materials in ULSI technology is related to their degradation during different technological processes such as thermal annealing, plasma etch and resist strip, cleaning and chemical mechanical polishing etc. Removal of carbon containing hydrophobic groups and further adsorption of water and other polar molecules significantly increases the effective dielectric constant of low-k films [1]. Degree and depth of such damages increase with pore size and degree of their interconnectivity. Therefore, ultra low-k materials, which have high porosity and relatively large pore size, suffer much more than microporous materials [2, 3]. The depth and profile of the carbon depletion are normally evaluated using various analytical techniques such as Fourier Transform Infrared Spectroscopy (FTIR), X-ray Photoelectron Spectroscopy (XPS), Time-of-Flight Secondary Ions Mass spectroscopy (TOF-SIMS), Energy Filtered Transmission Electron Microscope (EFTEM) etc. [1]. It is assumed that the carbon depletion is directly responsible for the hydrophilicity and moisture adsorption, which finally defines effective dielectric constant of the film. The Si-O-Si backbone of hybrid low-k materials renders the matrix mechanically fragile and susceptible to stress-corrosion cracking in moist environments [4]. Increasing hydrophilicity caused by plasma processing makes this problem even more challenging.

In this paper, we present the basic ideas of recently developed method for evaluation of hydrophilic properties of low-k materials [5] and experimental results related to evaluation of 
thermal and plasma damage. Possibility of effective application of this method for quantitative evaluation of plasma damage has been demonstrated. Comparative evaluation showed that degree of hydrophilisation and depth of plasma damage has qualitative agreement with carbon depletion determined by Spectroscopic Ellipsometry, HF dip test and TOF SIMS.

Most of data reported in this paper were obtained with Spin-on-Glass (SOG) low-k films damaged by thermal annealing and etch/strip plasma.

\section{EXPERIMENTAL DETAILS}

The basic ideas of this method (water EP) are similar to Ellipsometric Porosimetry (EP) [6, 7]. EP set-up with a water source allows changing of vapor pressure from $10^{-3}$ to saturated ones $\left(\approx 20\right.$ torr). Amount of the adsorbed water $\left(\mathrm{C}_{\mathrm{wat}}\right)$ is calculated from change of refractive indices happening during the water adsorption by using Lorenz-Lorentz equation [6]:

$$
C_{\text {wat }}(\%)=100 \cdot \frac{V_{\text {water }}}{V_{\text {film }}}=100 \cdot\left[\left(\frac{n^{2}-1}{n^{2}+2}\right)-\left(\frac{n_{\text {min }}^{2}-1}{n_{\text {min }}^{2}+2}\right)\right] /\left(\frac{n_{\text {wat }}^{2}-1}{n_{\text {wat }}^{2}+2}\right)
$$

where $V_{\text {water }}$ and $V_{\text {film }}$ are volumes of adsorbed water and low-k film, respectively. $n$ is refractive index of the film with adsorbed water, $n_{\min }$ and $n_{\text {wat }}$ are refractive indices of the film with empty pores and liquid water, respectively. Amount of the adsorbed water in pristine low-k films reflects concentration of active centers like isolated hydroxyl groups. Technological damage increases number of the active centers and starting from a certain concentration, the adsorbed water forms continuous layer. As soon as it happens, the bulk condensation of water occurs [8]. Adsorption isotherms measured in the wide range of water vapor pressure allow characterization of hydrophilic properties that normally are quantified through the contact angle. This sort of analysis is based on the following ideas.

In EP, determination of the pore radius is based on the Kelvin equation that correlates the critical pressure $(P)$ when happens condensation, with pore radius $r$.

$\ln \left(\frac{P}{P_{0}}\right)=-\cos \theta * \frac{\gamma V_{L}}{R T} *\left(\frac{2}{r}\right)$

where $\gamma$ and $V_{L}$ are surface tension and molar volume of the liquid adsorptive, respectively. $\theta$ is the contact angle of the adsorptive. The adsorptive condenses in the pores at pressure $P$. To simplify the analysis and exclude possible uncertainties, special adsorptives with $\theta=0$ are used. Number of such adsorptives is limited: the most traditional one is nitrogen. Toluene and benzene show appropriate properties for the room temperature porosimetry $[7,9,10]$. If the contact angle is not equal to Zero, the Kelvin equation gives $r_{\text {eff }}=r / \cos \theta$. Therefore, comparison of the real pore radius obtained with adsorptive with zero contact angle (toluene: $r_{\text {tol }}$ ) with effective pore radius obtained by water adsorption ( $\mathrm{r}_{\mathrm{wat}}$ ) will allow us calculating water contact angle as $\cos \theta_{\mathrm{w}}$ $=\mathrm{r}_{\text {tol }} / \mathrm{r}_{\mathrm{wat}}$. The contact angle is a measure of surface energy: $\gamma_{S V}-\gamma_{S L}=\gamma_{L V} \cos \theta_{W}$, where $\gamma_{L V}$ 
is surface energy of liquid, $\theta_{w}$ is water contact angle. Surface energy of solid $\left(\gamma_{S V}\right)$ and energy of interface $\left(\gamma_{S L}\right)$ are in the left side. The calculated value of the contact angle $\left(\theta_{w}\right)$ is a unique characteristic reflecting internal surface energy of a damaged low-k material. To our best knowledge, no other technique as simple as EP allows one to measure it. Moreover, these results directly reflect hydrophilic properties that are important for the k-value.

SOG low-k films with initial porosity close to $30 \%$ and $50 \%$ were used in this work. The films were annealed up to $800^{\circ} \mathrm{C}$ to establish threshold of their thermal stability. In addition to “water EP”, they also were evaluated by FTIR, Hg-probe etc. [11].

\section{RESULTS AND DISCUSSION}

Thermal degradation of low-k materials is accompanied with change of thickness, porosity, composition and dielectric constant [11]. Here we will analyze change of hydrophilicity as a measure of degree of damage/degradation.
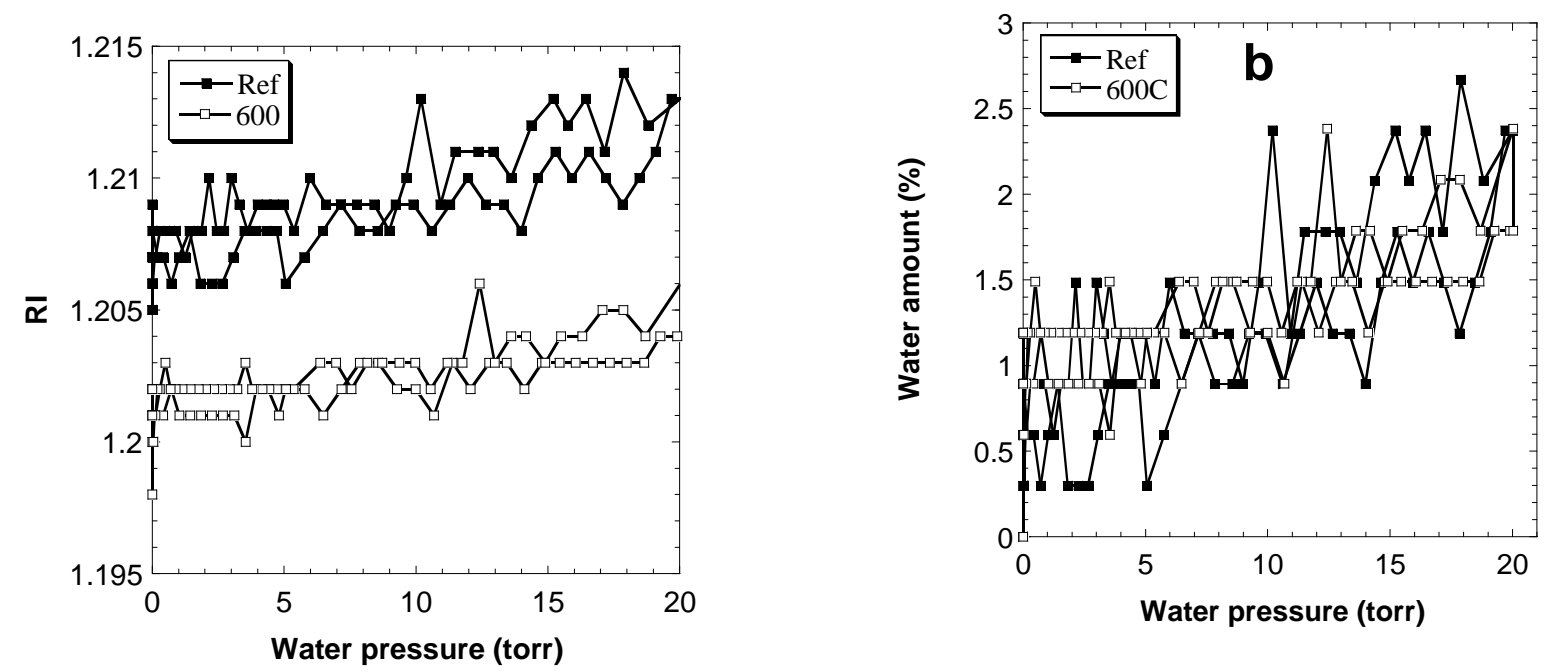

Fig.1. Change of refractive index (a) and hydrophilicity (b) after annealing at $600^{\circ} \mathrm{C}$. 


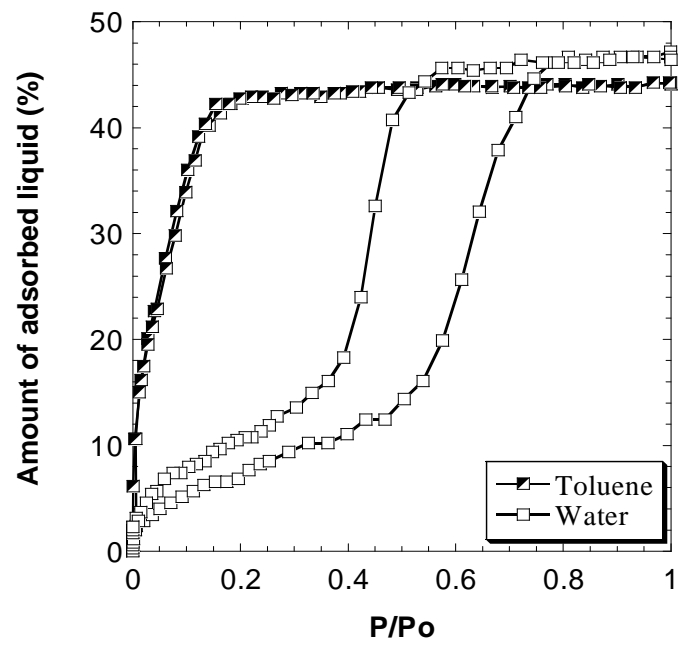

Fig. 2. Adsorption/desorption isotherms of toluene and water in low-k film annealed at $800 \mathrm{C}$.

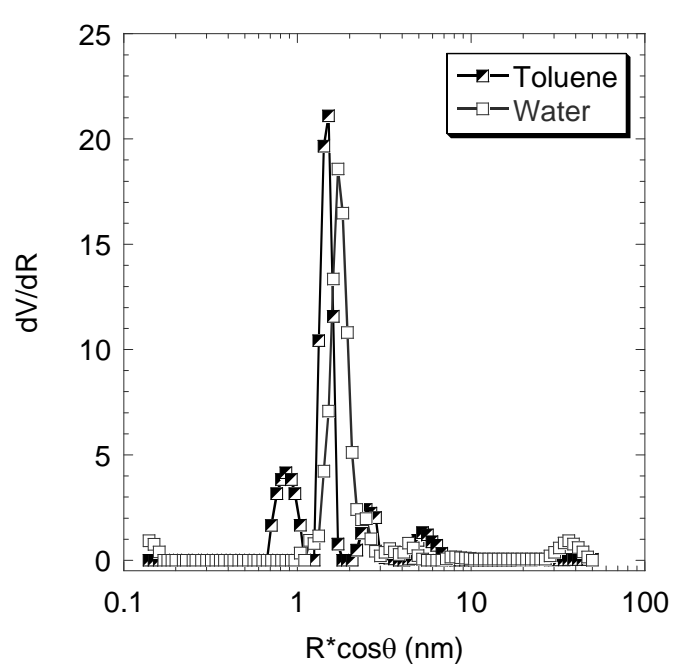

Fig.3. Pore radius distribution calculated from the data presented in Fig.3.

Figure 1 shows change of refractive index and water adsorption isotherms in pristine film and annealed at $600^{\circ} \mathrm{C}$. Pristine low-k materials are hydrophobic and amount of the adsorbed moisture at $100 \%$ of humidity do no exceed few percents in respects to the film volume. Stability of pristine low-k films was very good: no change of hydrophobicity was observed after several months. It is interesting that the low-k materials with improved mechanical properties often are more hydrophilic than others. The reason is, probably, that the improvement of mechanical properties needs increase of degree of cross-linkage that requires decrease of concentration of hydrophobic groups like $\mathrm{CH}_{3}$. One can see that that the films annealed at $600^{\circ} \mathrm{C}$ shows a small decrease of refractive index, which correlate with decrease of dielectric constant [11]. However, their ability to adsorb moisture (hydrophilicity) is the same. These data suggest that no degradation of low-k films occurs at $600 \mathrm{C}$ but insignificant decrease of dielectric function is related to removal of remaining porogen.

Figure 2 demonstrates SOG low-k films annealed at $800^{\circ} \mathrm{C}$. The amount of adsorbed water in the film annealed at $800^{\circ} \mathrm{C}$ was almost equal to the amount of adsorbed toluene ( $45 \%$ for toluene and $48 \%$ for water). That means very good wetting of internal pore surface by water and bulk water condensations happens in the pores. Small difference in porosity is probably related to smaller size of water molecules, which make possible to fill micropores not accessible for toluene. The pore radius calculated from the desorption branch of water adsorption is close to radius calculated from toluene adsorption (Figure 3), which proves that the contact angles of both toluene and water are close to zero. 

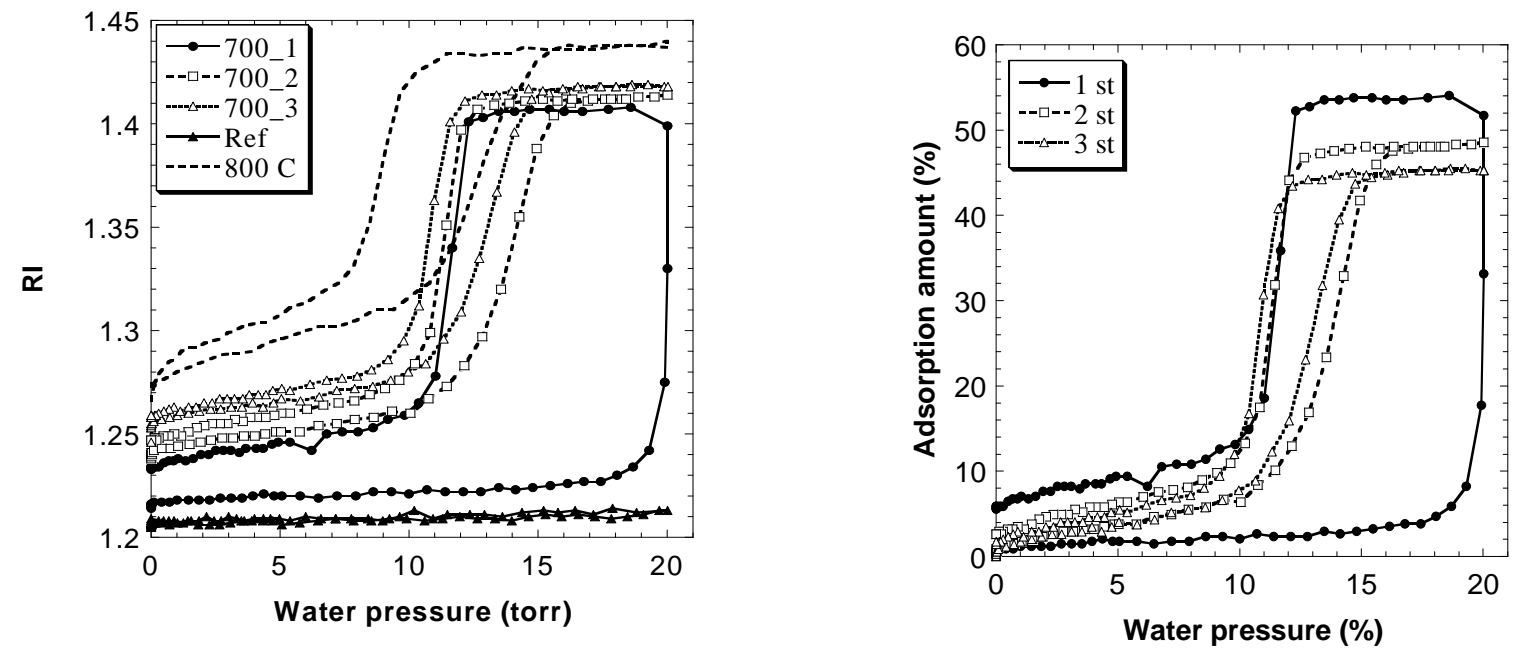

Fig.4. Change of refractive index (a) and calculated amount of adsorbed water (b) as a result of consecutive water adsorption.

The film annealed at $700^{\circ} \mathrm{C}$ shows more complicated behavior (Fig.4). This film shows almost hydrophobic behavior in the beginning and during the first adsorption cycle (700_1 in Fig.4). However, very fast condensation with complete pores filling happens at the water pressure close to 20 Torr. Desorption of the condensed water occurs at significantly lower pressure close to 10 Torr. However, the desorption pressure is still higher in comparison with the film annealed at $800 \mathrm{C}$. The internal contact angle calculated from the desoprtion curve is equal to $30^{\circ}$, which is in agreement with the contact angle measured on external surface. During the following (additional) adsorption cycles water condenses at much lower pressure (between 13-15 Torr). The desorption pressure is almost the same for the first and following adsorption cycles. An interesting and important feature demonstrates change of refractive index of the film in vacuum after complete desorption (initial refractive index). Each adsorption cycle increases this value that suggests that the moisture induced degradation includes increase of the initial refractive index, which is probably related to the densification of low-k film (Fig.4a). Degradation is also observed during the air storage.

A possible mechanism of such a phenomenon can be based on a model similar to proposed by Progogine and Fripiat [12]. Thermal anneal generate certain amount of hydroxyl groups able to adsorb water. The water molecules adsorbed on isolated hydroxyl ( $\mathrm{SiOH})$ groups react with adjacent siloxane surface to form two new SiOH groups. As a result, the hydrophilisation process gets autocatalytic character.

\section{Conclusions}

Adsorption of water vapors allows examination of hydrophilic properties of porous low-k dielectric films. This method can be used for comparative characterization of different low-k films and also degree of technological damage including unique possibility to measure contact angle (surface energy) of internal surface of porous films. The measured internal contact angle has a reasonable agreement with contact angle of the damaged top surface. 


\section{References.}

1. K. Maex, M. R. Baklanov, D. Shamiryan, F. Iacopi, S. Brongersma and Z. Sh. Yanovitskaya, J. Appl. Phys. 93, 8793 (2003).

2. M. R. Baklanov, Y. Travaly, Q. T. Le, D. Shamiryan and S. Vanhaelemeersch, In: Silicon Nitride, Silicon Dioxide, Thin Insulating Films and Other Emerging Dielectrics YIII", Ed. R.E.Sah, M. J. Deen, J. F. Zhang, J. Yota, Y. Kamakura. ECS, PV 2005-01, pp. 179-198.

3. D. Shamiryan, M. R. Baklanov, S. Vanhaelemeersch, and K. Maex, J. Vac. Sci. Technol. A20, 1923 (2002).

4. E. P. Guyer and R. H. Dauskardt. J. Mater. Res., 20, 680 (2005).

5. M. R. Baklanov, K. P. Mogilnikov and T. Q. Le, Microelectronic Eng., to be published.

6. M. R. Baklanov, K. P. Mogilnikov, V. G. Polovinkin and F. N. Dultsev, J.Vac.Sci.Technol. B18, 1385 (2000).

| 7. M. R. Baklanov and K. P. Mogilnikov, Microelectronic Eng., 64 (1/4), 335(2002).

8. T. Ohba, H. Kanoh, and K. Kaneko. Nanoletters, 5(2), 227 (2005).

9. W. Wu, W. E. Wallace, E. Lin, G. W. Lynn, C. J. Glinka, R. T. Ryan and H. Ho, J. Appl. Phys., 87, 1193 (2000).

10. R. C. Hedden, H-J. Lee, C. L. Soles and B. J. Bauer. Langmuir, 20, 6658 (2004).

11. S.Demuynck, E.Hong, Q.T.Le, M.R.Baklanov, M.Van Hove and J.M.Lee. AMC 2005 Proceedings, pp.12-13, 2005.

12. M. Prigogine and J. J. Fripiat. Bull. Soc. Roy. Sci. Liege, 7-10, 449, (1974). 